\title{
Über die chemisch-quantitative Zusammensetzung der Staßfurter Salzablagerungen.
}

Von

M. Rózsa.

Vergleicht man den organischen Aufbau des StaBfurter Salzlagers mit der sukzessiven Reihenfolge der Salzausscheidungen eintrocknenden Meerwassers, so läßt derselbe den Ursprung aus Meerwasser deutlich erkennen. $\mathrm{Zu}$ dem verhältnismäBig ruhigen Stadium eines kontinuierlichen Eintrocknungsprozesses der Binnenseen und demzufolge zur Entstehung des organischen Schichtenaufbaues der Salzablagerungen kam es erst in der Zechsteinperiode. DaB aber die Bildungsneigung bereits in früheren Formationen vorhanden war beweist der Umstand, daß Absätze des schwefelsauren Calciums und des Dolomits sowohl in der deronischen Grauwacke, als auch in den versteinerungsleeren Schiefern vorhanden sind.

GroBe Schwierigkeit bereitet jedoch bei dieser Auffassung die Mächtigkeit der Steinsalzlagerstätten. Wenn man auch die gewaltigen Dimensionen bis zu $1000 \mathrm{~m}$ Steinsalz bei Wansleben und bis zu $1200 \mathrm{~m}$ bei Sperenberg auf spätere tektonische Einwirkungen zurückführen kann, so lassen sich die tatsäcblich mehrere hundert Meter mächtigen Steinsalzlager durch den in einem abgeschlossenen Becken vorgegangenen einfachen VerdunstungsprozeB doch kaum erklären.

Auf Grund der Angaben Usratros müBte im StaBfurter Becken zur Zeit der Entstehung der kieseritischen Zone die Tiefe der vorhandenen Mutterlauge - bei $400 \mathrm{~m}$ bereits stattgefundener Salzablagerung - noch immer über $500 \mathrm{~m}$ betragen haben. Dies würde bei der Annabme von ausschlieBlich in vertikaler Richtung wirkenden Sättigungsströmen einer ursprünglichen Seetiefe von ungefähr $32.000 \mathrm{~m}$ entsprechen.

Bei Berücksichtigung des quantitativen Verhältnisses, welches zwischen den Salzen schwefelsaures Calcium und Chlornatrium im heutigen Meerwasser besteht, wäre unter einem Steinsalzlager von $400 \mathrm{~m}$ Stärke ein Anhydritlager von kaum $18 \mathrm{~m}$ zu erwarten, wenn

Z. anorg. Chem. Bd. 90. 
sich auch die ganze Menge des schwefelsauren Calciums noch vor der Steinsalzablagerung ausgeschieden hätte; ein Fall, der doch gewiB nicht stattgefunden hat. Tatsächlich befindet sich aber unter dem Steinsalze ein ca. 50-70 m starkes Anhydritlager, welchem ein ca. $1400 \mathrm{~m}$ starkes Steinsalzlager entsprechen würde, woza Seetiefen von über $100.000 \mathrm{~m}$ nötig wären. Eine Erklärung über die außerordentliche Mächtigkeit der Salalagerstätten gab Ochsenrus durch die Aufstellung seiner bekannten Barrenhypothese, welche sich hauptsächlich auf die Verhältnisse des Adschi-Darja-Busens stützt. Diesen Busen schlieBt nämlich eine Barre fast vollständig rom Kaspisee ab, und gewäbrt durch eine Kara Bugas (schwarzer Schlund) genannte freie Stelle den Zufluß von Seewasser nur in solch geringem MaBe, daB infolge des herrschenden Steppenklimas eine starke Konzentrierung der Salzlösung in demselben stattfinden kann. Ein Teil der sich ansammelnden Mutterlauge gelangt wieder in den Kaspisee zurück, an tieferen Stellen des Busens sinkt jedoch die konzentrierte Lösung zu Boden und es tritt ein Sättigungsprozeb ein.

Nach der Ochseniusschen Hypothese bildeten sich demnach mächtige Steinsalzlager hauptsächlich in solchen Binnenseen, die durch eine Landesbarre vom Ozean mehr oder weniger abgetrennt waren. Der Umstand, daB in den Steinsalzablagerungen die Kalisalze selten vorhanden sind, wird mit Hilfe dieser Hypothese dadurch erklärt, daß die Absätze der Binnenseen schließlich das Niveau der Barrenhöhe erreichten, wodurch infolge umgekehrter Strömungen die kalireichen Mutterlaugen in den Ozean zurückHließen muBten. Ein Zurückbleiben dieser Laugen konnte demnach nur infolge jener tektonischen Vorgänge stattfinden, welche infolge der Erhöhung der Barre einen Abschluß des Busens vom Ozean verursachten.

So verlockend auch die von Ochsenids aufgestellte Hypothese klingt und uns über manche Schwierigkeiten des Salzproblemes hinüberhilft, kann ein solch organisch regelmäBig aufgebautes Steinsalzlager, mit so geringen Spuren der Carbonate, in solcher Reinheit und mit solch regelmäBigem Wechsel, der Anhydrit- und Steinsalzschichten, wie dasselbe in StaBfurt vorkommt, durch einen kontinuierlichen ZufluB des Meerwassers doch nicht entstanden sein. ${ }^{1}$ Nach den Daten der Tiefbohrungen befinden sich im älteren

1 J. Walther, Lehrbuch der Geologie von Deutschland, S. 94. 
Anhydrit bituminöse Dolomitbänke und durchgehende mehrere Meter starke Steinsalzbänke und liefern einen Beweis dafür, daB die $\mathrm{Zu}$ sammensetzung der eintrocknenden Lauge sich im Anfangsstadium, der Gips- und Steinsalzausscheidung durch sporadische Überlagerungen von Laugen rückgängig gestalten konnte, weshalb schon in diesem frühen Eintrocknungsstadium der Zechsteinseen mit dem Ozean keine Verbindung bestanden hat. ${ }^{1}$

Andererseits vollzog sich die Zusammenschrumpfung der eintrocknenden Lauge tieferer Beckenteile von großen Flächen, da Salzablagerungen - wenn auch stellenweise durch spätere Auslaugungen verschwunden - von den russischen Grenzen bis nach Holland hinein, und von den Nord- und Ostseen bis an den FuB der deutschen Mittelgebirge fast überall vorzufinden sind.

Diese Umstände, verbunden mit stattgefundenen Lokalisationen der sättigenden Konzentrationsströme in den tieferen Beckenteilen, ergeben die natürliche Erklärung sowohl über die große Mächtigkeit des Anhydritlagers, als auch über jene des Steinsalzes.

Wie bereits erwähnt, ist die Mächtigkeit des Stabfurter Steinsalzlagers dem Anhydritlager gegenüber eine viel zu geringe, denn einem Anhydritlager von über $50 \mathrm{~m}$ Stärke müBte ein mindestens $1200 \mathrm{~m}$ starkes Steinsalzlager entsprechen. Es fand demnach während der Steinsalzablagerung im Zechsteinsee kein Zufluß von Meerwasser statt, sondern im Gegenteil, ein bedeutender Teil der Lauge floB infolge tektonischer Bodengestaltungen vom Zechsteinsee $a b$

Bedeutende Überlagerungen von Laugen verschiedenen Eintrocknungsgrades - entstanden infolge tektonischer Bodengestaltungen - lieBen sich im Stadium der Kalisalzausscheidungen, ebenfalls feststellen. ${ }^{2}$

In der anhydritischen Steinsalzzone sollten nach den Versuchsdaten $\nabla_{\triangle N}{ }^{\prime} T$ Horrs, beim Verlauf der bei $25^{\circ}$ vor sich gehenden Eintrocknung $1.77 \%$ Anhydrit, auf $98.23 \%$ Steinsalz entfallen. Die Hauptmasse des schwefelsauren Calciums schied sich bereits vor der Steinsalzbildung aus, und zwar hauptsächlich als Gips, da der Dehydratationsgrad von $25^{\circ}$ erst bei späterer Konzentrationszunahme eintritt. Hätte der AusscheidungsprozeB dieses schwefel-

1 Zeitschr. f. Elektrochem. u. angew. Ch. 1913, Heft 3, und Über den organischen Aufbau der StaBfurter Salzablagerungen (Berlin, R. Friedländer \& Sohn 1914). Vgl. auch Kali 1913, Heft 10.

\& Über die posthumen Umwandlungen der Kali- und Magnesiasalze in den Salzablagerungen der Werragegend [Z. anorg. Chem. 88 (1914), 321-332]. 
sauren Calciums teilweise auch bei den höheren Temperaturen der Dehydratation stattgefunden, so müBte in dem anhydritischen Steinsalze noch weniger Anhydrit vorhanden sein, als bei $25^{\circ}$ angegeben wird.

Tatsächlich entfallen in der anhydritischen Steinsalzzone $4.2 \%$ Anhydrit auf $95.8 \%$ Steinsalz. Diese Zusammensetzung entspricht annähernd jenem Verhältnisse, in welchem die Salze $\mathrm{CaSO}_{4}$ und $\mathrm{NaCl}$ im Meerwasser vorkommen. Nun wurde aus dieser Tatsache eine Bestätigung der OcHsenrusschen Barrentheorie abgeleitet und behauptet, daß zwei solche zusammengehörende Schichten den Eintrocknungsprodukten jener Menge des Ozeanwassers entsprechen, welche einem jährlichen Zufluß über die hypothetische Barre gleichkommt.

Aus dem Umstande, daB die Löslichkeit des schwefelsauren Calciums über der Dehydratationstemperatur abnimmt, wurde ferner der SchluB abgeleitet, daB die Ausscheidung des Anhydrits in den Sommermonaten geschah, während die $Z$ wischenlagerungen von Steinsalz sich während der übrigen Jahreszeiten bildeten.

Aus physikalisch-chemischen Gesichtspunkten muB nun die Entstehung der periodischen Anhydritschichten in der Weise erklärt werden, daß nach der Bildung des Gips-Anhydrithauptlagers, die Konzentration und die Gleichgewichtszustände der eintrocknenden Lauge schlieBlich das Stadium der konstanten Lösung von schwefelsaurem Calcium und von Chlornatrium erreichten, und unter der Debydratationstemperatur des Gipses sich Steinsalz und geringe Mengen ron Gips - später infolge von Temperaturerhöhungen des Bodens in Anbydrit umgewandelt - gemeinschaftlich ausscheiden konnten. Stieg nun in der warmen Jahreszeit die Temperatur der Lauge tuber die Dehydratationsgrenze des Gipses (ungef ähr $25^{\circ}$ bei dieser Konzentration und Zusammensetzung), so schied sich infolge der Löslichkeitsabnahme des entstandenen Anbydrits der Überschuß desselben aus, und bildete die anhydritischen Jahresschichten. ${ }^{1}$

Nach der Ausfällung des überschüssigen Anbydrits, jedoch während der warmen Jahreszeit, erreichte die Lösung auch den Gleichgewichtszustand der gemeinschaftlichen Ausscheidung von Steinsalz und von Anhydrit. Während dieser Zeit, also noch in der warmen Jahreszeit schied sich daher die Hauptmenge des Stein-

1 Über den organischen Aufbau der StaBfurter Salaablage. rungen, S. 11 . 
salzes aus, mit minimaler Beimengung von Anhydrit. Bei Abnahme der Lufttemperatur nahm auch die Temperatur der Lauge ab, die Löslichkeit des Anhydrids aber bis zum Hydratationsgrade zu, demzufolge sich fast reines Steinsala ausschied. Bei weiterer Abnahme der Temperatur, bis unter den Dehydratationsgrad des schwefel. sauren Calciums, nimmt sowohl die Löslichkeit des Gipses, als auch jene des Steinsalzes ab, weshalb bei diesen Temperaturen sich ebenfalls Steinsalz ausschied, und zwar im Zusammenhange mit den Temperaturverhältnissen und je nach den Tiefen, in welchen die Lokalisationen der sättigenden Konzentrationsströme stattgefunden haben, mit variierenden geringen Mengen des Gipses.

Während der kühlen, regnerischen Witterung konnten sich infolge der Verlangsamung, eventuell infolge des temporären Ausbleibens des Sättigungsprozesses relativ gröBere Mengen schwebender Ton- und Sandteilchen aus der Lauge ablagern. ${ }^{1}$ Erwärmten sich aber in der nachfolgenden neuen Periode der trockenen, warmen Jahreszeit die oberen Seeschichten, so kam es infolge der Verschiebung des Gleichgewichtszustandes zwischen dem Chlornatrium und dem schwefelsauren Calcium, bei über der Dehydratationsgrenze des Gipses liegenden Temperaturen, zu neuer Differenzierung der Ausscheidung beider Verbindungen und demnach zur isolierten Ausscheidung des überschüssigen Anhydrits. Mit dieser unmittelbaren Aufeinanderfolge der Ausscheidungen bzw. der Jahreszeiten hängt offenbar das gesteigerte Vorkommen von Ton, Sand und Bitumen in den periodischen Anhydritschichten zusammen.

Die Resultate meiner diesbezüglichen Analysen sind in der folgenden Tabelle zusammengefaBt.

Die Mächtigkeit des mit dem neuen Meereseinbruch zusammenhängenden jüngeren Hauptanhydrits gegenüber der Mächtigkeit des jüngeren Steinsalzes im Stabfurter Lager, deutet ebenfalls auf den teilweisen AbfluB der auf Steinsalz gesättigten Lauge, welcher AbfluB diesmal infolge eines neueren Meereseinbruches erfolgte.

Die tonreichen Jahresschnüre des jüngeren Steinsalzes liegen weiter entfernt voneinander, als die periodischen Anhydritschnüre im älteren Steinsalz, demzufolge auch die zwischengelagerten Steinsalzschichten in demselben bedeutend stärker sind. Die durch-

1 Staubtürme haben jedenfalls zur Differenzierung des Ton- und Sandgehaltes beigetragen. 


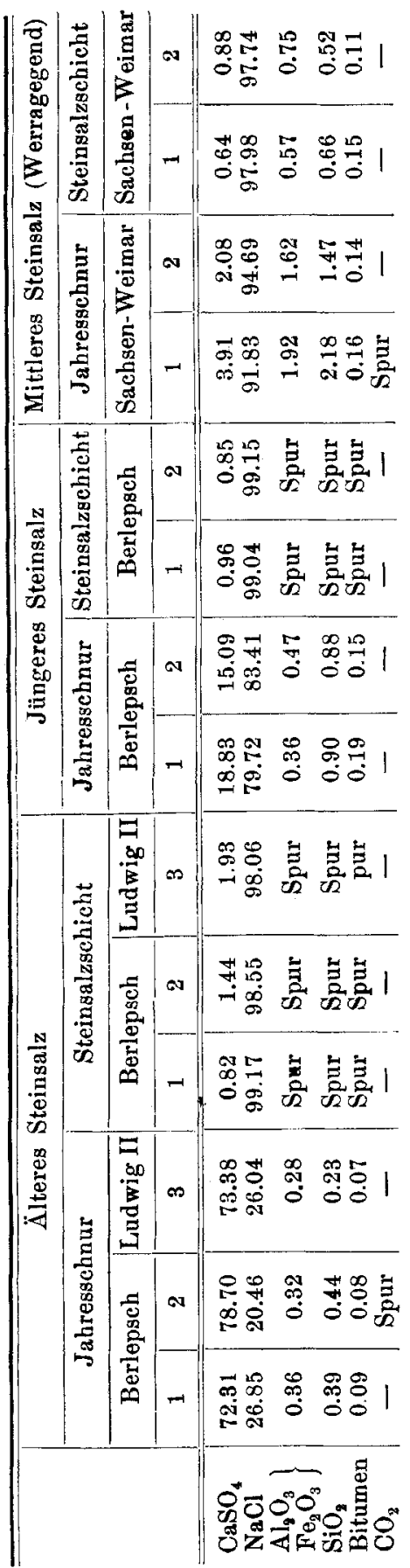

schnittliche Salzausscheidung zwischen den Perioden derselben Jahreszeit war demnach zur Bildungszeit des jüngeren Steinsalzes eine viel gröBere, weshalb auch die Dauer der warmen, trockenen Jahreszeit gegenüber der kühleren Witterung der älteren Salzausscheidungsperiode eine bedeutende Zunahme erhalten muBte.

Da das überflutende Meerwasser gröBere Mengen der chlormagnesiareichen Laugen in sich aufnahm, und stellenweise bedeutende Mengen von abgelagertem Carnallit auflöste, ${ }^{1}$ so war auch die Möglichkeit nicht ausgeschlossen, da $B$ bei dieser Zusammensetzung der Lauge und bei den herrschenden Temperaturen des Eintrocknungsprozesses die Hauptmenge des jüngeren Hauptanhydrits sich als solcher ausschied, weshalb sich vor der Steinsalzausscheidung relativ mehr Anhydrit ablagern konnte, als im älteren Lager, und zur Zeit der Steinsalzausscheidung die Lauge geringere Mengen von schwefelsaurem Calcium enthielt. Infolge der kürzeren

1 Hiermit verbunden läBt sich die Eventualität der primären Ausscheidung von Sylvin im jüngeren Lager erklären. Durch aufwärts strebende chlormagnesiareichen Laugen des älteren Lagers wurde derselbe stellenweise zu Carnallit umgewandelt. In den chlormagnesiareichen tieferen Beckenteilen schied sich jedoch der Carnallit auch primär aus (Z. anorg. Chem. 88 (1914), 323. 
Dauer der kühlen Jahreszeit, welche durch die erwähnteVeränderung der klimatischen Verhältnisse verursacht wurde, ist die Differenzierung dieses zurückgebliebenen schwefelsauren Calciums im jüngeren Steinsalzlager von sehr geringem Maße. Bei der kurzen Dauer war nämlich diese küble Jahreszeit gleichzeitig eine Regenzeit, so daB es in dieser Periode za Ausscheidungen kaum gekommen sein konnte, weshalb auch in Anbetracht der geringen Mengen des in der Lösung zurückgebliebenen schwefelsauren Calciums - jene Verschiebungen des Gleichgewichtszustandes zwischen dem Chlornatrium und dem schwefelsauren Calcium, welche während der Ausscheidung des älteren Steinsalzes zur Übersättigung des Anhydritgehaltes führten, nur in sehr geringem MaBe auftraten. Infolge dieser Umstände ist auch der Polyhalitgehalt des jüngeren Steinsalzes ein relativ geringer. ${ }^{1}$

Es bleibt noch die Aufklärung jenes Umstandes übrig, wober der überaus große Anhydritgehalt des älteren Steinsalzes in Staßfurt herstammt.

Die während der Regenzeit stattgefundene Vermischung der von den bereits trockengelegten Gebieten zuflieBenden Laugen mit der Mutterlauge konnte jedenfalls zur Sättigung in den oberen Seeschichten beitragen. Die Mengenverhältnisse und die RegelmäBigkeit der periodischen Anhydritschichten schließen jedoch die Annahme, daB die quantitativen Abweichungen dieser rezidiven Anbydritbildung ausschlieBlich auf diese Zuflüsse zurückzuführen seien, gänzlich aus. Nach den Lagerungsverhältnissen beurteilt, machte sich die Wirkung der Zufüsse zur Zeit der carnallitischen Schichtbildung, also im vorgeschrittenen Stadium der Laugeneinschrumpfung und bei relativ großem Zuwachs des Inundationsgebietes am meisten geltend.

Die quantitativen Abweichungen in der Zusammensetzung des anhydritischen Steinsalzes hängen mit stattgefundenen Lokalisationen der Konzentrationsströme zusammen, da die Bodenlauge spezifisch schwerer war als die Lauge der höheren Seeschichten, weshalb auch die während der warmen Jahreszeit stattgefundene Ausscheidung des Steinsalzes und des Anhydrits bis in gewisse Seetiefen lokalisiert blieb. Die Abküblung und die Dichtigkeitszunahme der oberen Schichten während der kühlen Jahreszeit verursachten nun, infolge der tiefer eindringenden Zirkulation des Salzwassers, eine partielle

1 Über den organischen Aufbau der StaBfurter Salzablagerungen, S. 33. 
Regeneration der Zusammensetzung in den oberen Seeschichten und trugen daher zu den quantitativen Abweichungen der periodisch auftretenden Rezidiv-Anhydritschichten bei.

Das periodische Auftreten von Kieseritschnüren in StaBfurt kann als sicherer Beweis der Annahme betrachtet werden, daB in den oberen Seuschichten Ausscheidungstemperaturen auch über $30^{\circ}$ geherrscht haben. Wenn auch infolge der erwähnten Lokalisationen und infolge der Trägheit der sättigenden Konzentrationsströme in den unteren Regionen der tieferen Seen gleichzeitig niedrigere Temperaturen vorausgesetzt werden, so verhinderte dieser Umstand die Ausscheidung von dehydratisierten Sulfaten aus den oberen Seeschichten doch nicht, wenn auch die Gleichgewichtszustände der kälteren Bodenlauge zeitweise ein Übergangsstadium der Rehydratation bei der Ablagerung verursachen konnten.

Deszendente Laugen, infolge der teilweisen Wiederauflösung von Salzlagern entstanden, konnten das schwerlösliche schwefelsaure Calcium in solch geringer Menge enthalten, daB es zum Gleichgewichtszustande der Differenzierung des letzteren überhaupt nicht kam.

Meine Untersuchungen 'über die Ablagerungen im Adschi DarjaBusen muBte ich infolge der kriegerischen Ereignisse leider unterbrechen. Sobald es jedoch möglich wird, gedenke ich dieselben weiter fortzusetzen.

\section{Zusammenfassung.}

Die von Ochsenros aufgestellte Barrentheorie, welche sich auf die Salzausscheidungen im Adschi Darja-Busen stützt, widerspricht den physikalisch-chemischen Bedingungen, die während der Entstehung des Anhydritlagers und des anhydritischen Steinsalzes in den Zechsteinbinnenseen bestanden haben.

Die während des Eintrocknungprozesses in den tieferen Beckenteilen von großen Flächen stattgefundene Zusammenschrumpfung isolierter Meereslaugen, und die infolge tektonischer Bodengestaltungen stattgefundenen Überlagerungen von Laugen, ergeben eine natürliche Erklärung der bedeutenden Schichtmächtigkeiten des Anhydrits und des Steinsalzes.

Die Entstehung der anhydritischen Jahresschichten und die quantitativen Verbältnisse der periodischen Differenzierungen zwischen dem Calciumsulfat und dem Chlornatrium wurden hauptsächlich durch die herrschenden klimatischen Verhältnisse und durch die er- 
Chem.-quantitat. Zusammensetzung der Staßfurter Salzablagerungen. 385 folgten Lokalisationen der sättigenden Konzentrationsströme verursacht.

Während der Ausscheidung des älteren Steinsalzes fand infolge tektonischer Bodengestaltungen rom StaBfurter Becken her ein bedeutender AbfluB - Flächenverbreiterung - der Zechsteinlauge statt.

Budapest, II. Städt. chem. Laboratorium, den 12. Oktober 1914.

Bei der Redaktion eingegangen am 2. November 1914. 